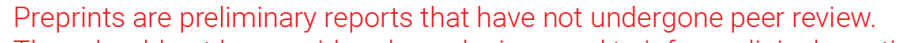 \\ They should not be considered conclusive, used to inform clinical practice, or referenced by the media as validated information. \\ Clinical Characteristics of Bacterial Spectrum and Antimicrobial Resistance in Urine and Stone Cultures From Patients With Kidney Stone Disease
}

Min Lei

Guangzhou Medical University https://orcid.org/0000-0002-8133-9174

Zheng Jiang

Guangzhou Medical University

Peng Xu

Guangzhou Medical University

Lingyue An

Guangzhou Medical University

Zhenglin Chang

Guangzhou Medical University

\section{Yuyan Zhang}

Guangzhou Institute of Geochemistry

\section{Shike Zhang}

Guangzhou Medical University Second Affiliated Hospital

\section{Tao Zeng}

Guangzhou Medical University

Shujue Li

Guangzhou Medical University

Hans-Göran Tiselius

Intervention and Technology,Karolinska Institute

\section{Guohua Zeng}

First Affiliated Hospital of Guangzhou Medical University

Wenqi Wu ( $\nabla$ wwqwml@163.com )

Guangzhou Medical University https://orcid.org/0000-0002-3923-3093

\section{Research}

Keywords: Urinary stones, Bacterial spectrum, Antibiotic resistance, Stone culture, Urine culture

Posted Date: November 15th, 2021

DOI: https://doi.org/10.21203/rs.3.rs-1056064/v1 
License: (c) (i) This work is licensed under a Creative Commons Attribution 4.0 International License. Read Full License 


\section{Abstract \\ Background}

It is important to understand the clinical characteristics of bacterial spectrum and antibiotic resistance of urine and stone pathogens for the prevention and treatment of urolithiasis and perioperative infection.

\section{Methods}

Consecutive patients with kidney stones treated by percutaneous nephrolithotomy (PCNL) from September 2016 to September 2018 were included. The bacterial species and antibiotic sensitivity test of the germs cultured from clean middle-stream urine and from stones intraoperatively were evaluated.

\section{Results}

In 1055 patients, the rate of positive bacterial test was higher in stones than in urine $(337,31.0 \%$ vs. 221 , $20.9 \%, p=0.016) .167(15.8 \%)$ patients had both positive urine culture (UC) and stone culture (SC), of which $137(82.0 \%)$ had identical bacteria in both cultures. In infection stone patients, the positive rate of bacteria was $34.7 \%(91 / 262)$ in urine and $52.3 \%$ (137/262) in stone, and in non-infection stone patients, was $16.4 \%(130 / 793)$ and $25.2 \%$ (200/793). The positive rate of SC in patients with different types of stones were higher than that of UC. E. coli was the most common organism not only in both UC $(54.3 \%$ ,120/221) and SC $(43.9 \%, 148 / 337)$ but also in urine and stones from patients with infection stones $(44.0 \%, 32.8 \%)$ or non-infection stones $(61.5 \%, 51.8 \%)$. Furthermore, the pathogens isolated from urine and stones showed high resistance to fluoroquinolones, ceftriaxone, cefazolin, cefuroxime, $\beta$-lactamases and sulfonamides (all resistance $>20 \%$ ).

\section{Conclusions}

The bacterial spectra demonstrated in stones and urine samples were significantly different. Positive SC was more commonly encountered than positive UC. Compared with non-infection stones, infection stones were accompanied by higher rates of positive tests in both cultures. The antibiotic resistance was comparable between bacteria in the two cultures. A combination of antibiotic sensitivity results in urine and stones might be a useful guide for selection of effective and appropriate treatment aiming at reduced problems with bacterial antibiotic resistance.

\section{Introduction}

Urolithiasis is one of the most common global diseases in urology and the incidence is gradually increasing $[1,2]$. The presence of urinary stones is often accompanied by urinary tract infections (UTI) 
and commonly associated with urine flow obstruction[3].This combination might promote the further development of stones $[4,5]$.

Stones can be divided into infectious stones and non-infectious stones by aetiology. According to the latest EAU guidelines, the ingredients of infectious stones include magnesium ammonium phosphate, highly-carbonated apatite and ammonium urate. Previous Chinese studies have shown that the most common pathogen in stone-related UTI is Escherichia coli, for which multiple drug-resistant subgroups account for a proportion as high as $80 \%[6]$. Consequently, these infections are very difficult to treat. Percutaneous nephrolithotomy (PCNL) is the most common method for surgical removal of kidney stones with diameters $>2 \mathrm{~cm}[7,8]$. Serious postoperative complications may follow PCNL which, if not treated in time may develop into urosepsis and life-threatening septic shock $[9,10]$. Perioperative infection complications might occur despite intensive preoperative antibiotic therapy based on microbiological analyses of urine [11].

Previous studies showed that results of mid-stream urine cultures from patients treated with PCNL were not sufficiently reliable in reflecting bacteria in renal pelvic urine or stones [12,13], especially in the presence of urinary tract obstruction. Moreover, both urine cultures (UC) and stone cultures (SC) were independent risk factors for urosepsis in patients with complex urinary stones. The simultaneous positive occurrence of bacteria in urine and stones was associated with an increased risk of postoperative infections compared with the situation when only a single positive culture was recorded [14].

In several studies it was only shown that the results of UC and SC were not always the same, Nevertheless, a comparison and analysis of the specific differences between the bacterial spectrum in stones and urines were not carried out. But it is necessary to understand the characteristics of urine and stone bacteriology and the degree of antibiotic sensitivity for adequate treatment of patients with urinary stones. In the current study, we retrospectively determined the bacterial spectrum and antibiotic sensitivity of SC and UC in patients with kidney stones. In addition, the positive results of SC and UC between infection stones and non-infection stones were compared to provide a scientific basis for a clinically rational selection of perioperative antibiotics.

\section{Patients And Methods}

\subsection{General information}

We included 1055 patients with kidney stones who consecutively had been subject to stone removal with PCNL in the first affiliated Hospital of Guangzhou Medical University from September 2016 to September 2018. Ethics Committee approval was obtained from the First Affiliated Hospital of Guangzhou Medical University. Informed consent was obtained from each patient.

\subsection{Collection method of the urine}


A clean mid-stream urine sample of about $10 \mathrm{~mL}$ was collected in a sterile test tube, and the samples were either cultured within 1 hour after collection, or stored at $4{ }^{\circ} \mathrm{C}$ and treated within 8 hours.

\subsection{Collection method of stones}

All patients were treated with pneumatic or ultrasonic lithotripsy during PCNL. After the core of the stone was removed with stone forceps, it was immediately immersed in sterile saline. Following elimination of impurities on the stone surface by washing 3 times, $10 \mathrm{~mL}$ of saline was added and the stone ground. The solution containing the ground stone was added to the nutritious broth and placed in the $37^{\circ} \mathrm{C}$ incubator for enrichment and culture. The process strictly followed aseptic principles.

\subsection{Bacterial culture and identification of strain}

The strains found in positive mid-stream urine cultures and stone cultures were identified by VITEK®2 automatic bacteria identification instrument. The bacterial antibiotic sensitivity test was performed with the KirbyBauer (Kmurb) method, and the results were interpreted according to the standards established by guidelines from the Clinical and Laboratory Standards Institute (CLSI).

VITEK®2 automatic microbiological identification instrument was purchased from French BioMérieux company. Antibacterial antibiotic sensitivity papers were purchased from British Oxoid company. Blood Agar and antibiotic sensitivity test medium Agar were purchased from Jiangmen Kailin Trading Co, Ltd.

\subsection{Statistical methods}

SPSS25.0 software was used for statistical analysis. The Chi-square test or Fisher's exact probability test was performed to detect differences between categorical variables. Significance was assumed at the 0.05 level.

\section{Results}

\subsection{The bacterial spectrum of urine and stones}

In the 1055 patients, positive stone cultures were recorded in 337 (31.9\%) compared with only 221 positive urine cultures (20.9\%). Table 1 shows the number and relative occurrence of common microorganisms in urine samples and stones. Emphasis was placed on the four most common types of kidney stones encountered in patients (Figure1a). Four of these stones were dominated by calcium oxalate, uric acid, cystine and infection components. The detection rate of bacteria in the stones was significantly higher than that in urines (Figure1b).

There were also statistically significant differences in the proportional pattern of antimicrobial spectra between UC and SC (Table 1). The most common microorganism observed in both cultures was E. coli, but its relative occurrence was significantly higher in UC than in SC (54.3\% vs 43.9\%; $p=0.016)$. Other frequently isolated pathogens in urine were Proteus mirabilis in 17 samples (7.7\%), Enterococcus faecalis in 15 samples (6.8\%) and Pseudomonas aeruginosa in 13 samples (5.9\%). The other common pathogens 
in stones were Proteus mirabilis in 41 (12.2\%), Pseudomonas aeruginosa in 18 (5.3\%) and Staphylococcus epidermidis in 14 (,4.2\%). The occurrence of Staphylococcus epidermidis in stones was higher than that in urine ( $0.9 \%$ vs $4.2 \% ; p=0.024)$, but Enterococcus faecalis was more common in urine $(6.8 \%$ vs $3.0 \% ; p=0.033)$.

In addition, 167 patients (15.8\%) had both positive urine and stone cultures (UC+ and SC+), of which the bacterial strains were identical in 137. Of these cultures 76 were found for infection stones, 18 of which $(23.7 \%)$ presented with different cultures between stone and urine. In the 91 patients with non-infection stones, the number of the patients with calcium oxalate stones, uric acid stones and cystine stones were 63,25 , and 3 , respectively. Twelve of these patients (13.2\%) had inconsistent UC and SC results.

We also found that 169 patients (16.0\%) with negative urine cultures had positive stone cultures (UC-, $\mathrm{SC}+$ ), the bacteria were mainly E. coli, Proteus mirabilis, Staphylococcus epidermidis, Klebsiella pneumoniae, and Pseudomonas aeruginosa. Moreover, the bacteria cultured from the $54(5.1 \%)$ of the patients with positive urine cultures but negative stone cultures (UC+, SC-), comprised E. coli, Klebsiella pneumoniae, Streptococcus agalactiae, Enterococcus faecalis and Pseudomonas aeruginosa.

We further separately analyzed the bacterial spectra in urine and stones from patients with infection and non-infection stones. These results are summarized in Tables 2 and 3. The most common bacteria was $E$. coli in infection stones and non-infection stones. Whether in urine or stone, Proteus mirabilis and Pseudomonas aeruginosa were significantly more common in patients with infection stones than in noninfection stones $(p<0.05)$. Among the 206 patients with infection stones, $34.7 \%$ had positive urine cultures, and $52.3 \%$ positive stone cultures. For 793 patients with non-infection stones, bacteria were detected in the urine of 130 patients (16.4\%) and in the stone of 200 patients (25.2\%) (Figure 2).

\subsection{The antimicrobial sensitivity of common bacteria in urine and stones}

\subsubsection{E. coli}

The drug resistance was almost the same for $E$. coli cultured from stones and urine (Figure 3a). Whether E. coli was isolated from stones or urine, entrains had high sensitivity for tigecycline, imipenem, meropenem, ertapenem, piperacillin/tazobactam, latamoxef, amikacin and nitrofurantoin.

The drug resistance for ampicillin was the highest $(84.5 \%, 80.9 \%)$, followed by Cefuroxime sodium (57.1\%,63.2\%) and Cefuroxime axetil (57.1\%,63.2\%). Among cephalosporins, resistance to the third and fourth generation of cephalosporins such as ceftazidime $(29.4 \%, 22.9 \%)$ and cefepime $(20.9 \%, 23.0 \%)$ were significantly lower than that of the first and second generations of cephalosporins, such as cefazolin $(64.8 \%, 56.1 \%)$ and cefuroxime $(61.0 \%, 54.4 \%)$.

\subsubsection{Proteus mirabilis}


Proteus mirabilis in urine and stones showed high sensitivity to carbapenem. Regarding beta-lactamase inhibitors, Proteus mirabilis was sensitive to cefoperazone / sulbactam, piperacillin / tazobactam and latamoxef. It was of note that Proteus mirabilis had high resistance patterns for nitrofurantoin $(100.0 \%, 97.0 \%)$ and tigecycline $(84.6 \%, 72.2 \%$; Figure $3 \mathrm{~b})$. They were resistant to some cephalosporins, such as cefazolin $(50.0 \%, 32.4 \%)$, cefuroxime sodium $(60.0 \%, 28.6 \%)$, cefuroxime axetil $(60.0 \%, 28.6 \%)$, ceftriaxone $(35.7 \%, 21.6 \%)$ and cefotaxime $(35.7 \%, 21.6 \%)$. In addition, they also had resistance to ampicillin (44.4\%,40.0\%), sulfamethoxazole (57.1\%,35.1\%) and ciprofloxacin $(22.2 \%, 26.7 \%)$.

\subsubsection{Pseudomonas aeruginosa}

Pseudomonas aeruginosa in urine and stones was resistant to most commonly used antibiotics, such as cefazolin, ceftriaxone, cefuroxime sodium, cefuroxime axetil and nitrofurantoin, ampicillin, sulfamethoxazole. These strains were completely sensitive to ceftazidime, cefepime, aztreonam, and polymyxin B. Pseudomonas aeruginosa in urine was more sensitive to beta-lactamase inhibitors such as piperacillin/tazobactam, cefoperazone/sulbactam and aztreonam (resistance $<20 \%$ ). These strains were more resistant to carbapenem and instance meropenem $(27.3 \%, 14.3 \%)$ than to aminoglycoside antibiotics such as tobramycin $(10.0 \%, 14.3 \%)$ gentamicin $(0.0 \%, 7.7 \%)$ and amikacin $(18.2 \%, 14.3 \%$; Figure3c).

\subsubsection{Enterococcus faecalis}

Enterococcus faecalis were completely resistant to sulfamethoxazole, clindamycin and quinupstin/dafopristin. Interestingly, Enterococcus faecalis in stones was more resistant to erythromycin than urine ( $75.0 \%$ vs $10.0 \% ; P=0.013)$. Whether Enterococcus faecalis in urine or stones, they all had high resistance to quinolones (Figure $3 \mathrm{~d}$ ).

\section{Discussion}

In this study, the proportion of calcium oxalate stones (55.2\%) was much lower than that reported in Zhang's article (77.5\%) [15], and the patients with uric acid stones were significantly more common. The explanation might be different dietary habits. It is possible that the patients in our study had eaten more foods with a high content urate/uric acid or urate precursors. Guangdong is a coastal area of South China and it was demonstrated that the composition of stones also was affected by geographical location, age and gender $[16,17]$. Interestingly, E. coli was the most common bacteria in both cultures. However, in the study by Paonessa [12], Staphylococcus was the most common pathogen in stones and urine. These findings may be attributed to differences in the distribution of urinary pathogens in various countries and regions. Consistent with the results of Walton and De Lorenzis et al [18,19], the rate of positive stone cultures (31.9\%) in our study was significantly higher than that of urine $(20.9 \%)$.

It needs to be mentioned that in the comparative analysis of antimicrobial spectra in stones and urine there were significant differences in the detection rate of E. coli, Enterococcus faecalis, and Staphylococcus epidermidis $(p<0.01)$. In 167 patients with both positive UC and SC, the non-coincidence 
rate between the two cultures was $18.0 \%$ (30 of 167). This was like previously reported findings [20]. Accordingly, the culture results of bacteria in urine did not fully reflect the findings in stones.

In mid-stream urine, the detection rate of Enterococcus faecalis was as high as $6.8 \%$, second only to the Proteus mirabilis (7.7\%). The increased number of invasive treatment modalities for stone removal such as the mini-percutaneous surgery, the extensive use of immunosuppressants, and the long-term and irregular use of antibiotics, have resulted in continuously increased antibiotic resistance for Enterococcus faecalis [21]. Furthermore, Enterococci usually have high biofilm-forming ability and strong adaptability to the environment, which makes them more and more difficult to eradicate $[22,23]$.

The spectrum of isolated uropathogens showed obvious differences between infection stones and noninfection stones. In urine, the antimicrobial spectrum of $E$. coli, the most commonly encountered bacteria in patients with infection and non-infection stones, was consistent with reports in the literature [24,25]. Moreover, we found that the frequency of $E$. coli was higher in patients with non-infection stones ( $p$ $=0.010)$, and also that the antimicrobial spectra were the same in stones $(p=0.001)$. Proteus mirabilis and Pseudomonas aeruginosa were significantly more common in infection stones than in non-infection stones, whether isolated from UC or SC $(p<0.01)$. This observation can be explained by the fact that infection stones mainly are caused by infection with urease-producing microorganisms. Proteus mirabilis is the main urease-producing microorganism [26].

Infection stones account for $10-20 \%$ of all human kidney stones, and their presence is associated with greater risk of renal tissue damage than is the case with non-infection stones. Infection stones also have a high recurrence rate [27]. Therefore, we also compared the antimicrobial bacterial spectra between patients with infection and non-infection stones. This was helpful for fully understanding the distribution of antimicrobial spectra in the different stone components and accordingly for the treatment strategy. Overall, the detection rate of bacteria in patients with infection stones was higher than that in patients with non-infection stones, irrespective of from which source the bacteria were isolated $(p<0.01$, Figure 2$)$. This observation proved that infection stones were caused by and associated with urinary tract infections. Non-infection stones were more related to the metabolism of oxalate and calcium. We have explained above that the choice of perioperative antibiotic regimen should be based not only on findings in mid-stream urine cultures, but also on the bacteriology in stone cultures.

E. coli and Proteus mirabilis were highly sensitive to carbapenem, followed by amikacin in aminoglycoside antibiotics. This observation was roughly consistent with the description in the China Antimicrobial Resistance Surveillance System (CARSS). But the disadvantage is the high cost of carbapenem antibiotics. Because of the wide antibacterial spectrum, strong antibacterial activity, and high stability to a variety of $\beta$-lactamases, carbapenem was rarely used for treatment of mild infections or for infection prevention. This antimicrobial agent is accordingly more suitable for severe infection in critically ill patients.

Pseudomonas aeruginosa was highly sensitive to polymyxin B, ceftazidime and cefepime, an observation that differed from that presented in the China Antimicrobial Resistance Surveillance System (CARSS). 
The reported sensitivity of Pseudomonas aeruginosa to the aminoglycoside antibiotics tobramycin, gentamicin and amikacin was $91.6 \%, 86.4 \%$ and $94.0 \%$, respectively, which was higher than that detected in stones analyzed in our study. Thus, urologists are requested not to underestimate the antimicrobial resistance of Pseudomonas aeruginosa. However, when using it as an empirical treatment for patients with urinary stones and systemic symptoms, it should be noted that amikacin has nephrotoxicity, ototoxicity, and other serious side effects.

It was worth mentioning that our study showed the drug resistance of Enterococcus faecalis to erythromycin in stones was significantly higher than that in urine, which to a certain extent explained that even if the use of antibiotics before operation could make urine culture negative, it was still difficult for us to treat the bacteria in stones. Extensive attention needs to be paid to bacteria isolated from stones because they may get directly into the blood circulation during lithotripsy and trigger postoperative infections [28]. Moreover, patients with positive cultures in both stones and urine were more likely to have fever [29].

Previous studies showed that UC and SC were independent risk factors for postoperative infection [14]. As mentioned above, we still found a high percentage of patients with positive stone culture despite negative urine culture. The reason may be that the use of antibiotics has not been so strict in China, and many patients can easily obtain antibiotics in pharmacies and hospitals. Before seeking medical advice, they may have carried out antibacterial treatment on their own. This situation may result in false negative urine cultures. Therefore, we suggest that in addition to routine urine culture before operation and antibacterial treatment according to the result, intraoperative stone culture always should be carried out. Similarly, we recommend doctors to fully realize and understand the history of antibiotic usage before stone treatment, it also is necessary to educate patients so that they correctly understand the dual nature of antibiotics and always to avoid the abuse of antibiotics.

The advantage of our study was that we not only described the characteristics of antimicrobial spectra of bacteria in urine and stones, but also emphasized the differences in UC and SC between infection stones and non-infection stones. These findings have not been observed in recent studies. Moreover, we analyzed the antimicrobial sensitivity of bacteria in urine and stones. These findings will be of great importance for the future perioperative antibacterial treatment of patients with urinary stones. We have emphasized that urologists never should underestimate the antimicrobial resistance of Pseudomonas aeruginosa and Enterococcus faecalis. The limitation of our report was that it only was a single-center study within a certain restricted region and our data mainly focused on patients with kidney stones. In the future, we also need to establish more antibiotic sensitivity data on common pathogens in urine and stones.

\section{Conclusion}

The antimicrobial spectra isolated in bacteria from stones and urine were significantly different. The occurrence of positive of SC was higher than that of positive UC. Patients with infection stones were 
much more likely to have positive cultures in both urine and stones than had patients with non-infection stones. The drug resistance was similar between bacteria in urine and stones. Combining the observations on drug resistance of bacteria in urine and stones should guide the use of effective and appropriate treatment regimens in association with active stone-removal.

\section{Abbreviations}

UC

urine culture

SC

stone culture

PCNL

percutaneous nephrolithotomy

\section{Declarations}

\section{Author contributions:}

ML: wrote the first draft of the manuscript. ZJ: analyzed the data. PX, LYA and ZLC: modified figures and tables. All authors contributed in collecting the data. YYZ, SKZ, TZ and SJL revised the manuscript. WQW, $\mathrm{GHZ}$ and Hans-Göran Tiselius assisted in reviewing and revising the manuscript. All authors read and approved the final manuscript.

Ethical approval: none.

Consent for publication: not applicable

\section{Availability of data and materials:}

The datasets used and analysed during the current study are available from the corresponding author on reasonable request.

\section{Competing interests:}

The authors declare that they have no competing interests.

\section{Acknowledgements:}

Not applicable.

\section{Funding:}

This work was supported by grants from National Natural Science Foundation of China (82070719, 81570633), Natural Science Foundation of Guangdong Province, China (2020A151501198), Research 
Project of Education Department of Guangdong province, China (2018KZDXM056), Special Project for the Construction of a High-level University of Guangzhou Medical University, China (201716007).

\section{References}

1. Scales CD, Jr., Smith AC, Hanley JM, Saigal CS, Urologic Diseases in America P. Prevalence of kidney stones in the United States. Eur Urol. 2012;62(1):160-5.

2. Zeng G, Mai Z, Xia S, Wang Z, Zhang K, Wang L, et al. Prevalence of kidney stones in China: an ultrasonography based cross-sectional study. BJU Int. 2017;120(1):109-16.

3. Heyns CF. Urinary tract infection associated with conditions causing urinary tract obstruction and stasis, excluding urolithiasis and neuropathic bladder. World J Urol. 2012;30(1):77-83.

4. Miano R, Germani S, Vespasiani G. Stones and urinary tract infections. Urol Int. 2007;79 Suppl 1:326.

5. Hsiao CY, Chen TH, Lee YC, Hsiao MC, Hung PH, Chen YY, et al. Urolithiasis Is a Risk Factor for Uroseptic Shock and Acute Kidney Injury in Patients With Urinary Tract Infection. Front Med (Lausanne). 2019;6:288.

6. Wang S, Zhang Y, Zhang X, Li J. An evaluation of multidrug-resistant (MDR) bacteria in patients with urinary stone disease: data from a high-volume stone management center. World J Urol. 2020;38(2):425-32.

7. Turk C, Petrik A, Sarica K, Seitz C, Skolarikos A, Straub M, et al. EAU Guidelines on Interventional Treatment for Urolithiasis. Eur Urol. 2016;69(3):475-82.

8. Rule AD, Lieske JC, Pais VM, Jr. Management of Kidney Stones in 2020. JAMA. 2020;323(19):1961-2.

9. Rizvi SAH, Hussain M, Askari SH, Hashmi A, Lal M, Zafar MN. Surgical outcomes of percutaneous nephrolithotomy in 3402 patients and results of stone analysis in 1559 patients. BJU Int. 2017;120(5):702-9.

10. Lewis S, Patel U. Major complications after percutaneous nephrostomy-lessons from a department audit. Clinical Radiology. 2004;59(2):171-9.

11. Wollin DA, Joyce AD, Gupta M, Wong MYC, Laguna P, Gravas S, et al. Antibiotic use and the prevention and management of infectious complications in stone disease. World $\mathrm{J}$ Urol. 2017;35(9):1369-79.

12. Paonessa JE, Gnessin E, Bhojani N, Williams JC, Jr., Lingeman JE. Preoperative Bladder Urine Culture as a Predictor of Intraoperative Stone Culture Results: Clinical Implications and Relationship to Stone Composition. J Urol. 2016;196(3):769-74.

13. Kreydin El, Eisner BH. Risk factors for sepsis after percutaneous renal stone surgery. Nat Rev Urol. 2013;10(10):598-605.

14. Chen D, Jiang C, Liang X, Zhong F, Huang J, Lin Y, et al. Early and rapid prediction of postoperative infections following percutaneous nephrolithotomy in patients with complex kidney stones. BJU Int. 2019;123(6):1041-7. 
15. Zhang D, Li S, Zhang Z, Li N, Yuan X, Jia Z, et al. Urinary stone composition analysis and clinical characterization of 1520 patients in central China. Sci Rep. 2021;11(1):6467.

16. Lieske JC, Rule AD, Krambeck AE, Williams JC, Bergstralh EJ, Mehta RA, et al. Stone composition as a function of age and sex. Clin J Am Soc Nephrol. 2014;9(12):2141-6.

17. Ye Z, Zeng G, Yang H, Li J, Tang K, Wang G, et al. The status and characteristics of urinary stone composition in China. BJU Int. 2020;125(6):801-9.

18. Walton-Diaz A, Vinay JI, Barahona J, Daels P, Gonzalez M, Hidalgo JP, et al. Concordance of renal stone culture: PMUC, RPUC, RSC and post-PCNL sepsis-a non-randomized prospective observation cohort study. Int Urol Nephrol. 2017;49(1):31-5.

19. De Lorenzis E, Boeri L, Gallioli A, Fontana M, Zanetti SP, Longo F, et al. Feasibility and relevance of urine culture during stone fragmentation in patients undergoing percutaneous nephrolithotomy and retrograde intrarenal surgery: a prospective study. World J Urol. 2021;39(6):1725-32.

20. Margel D, Ehrlich Y, Brown N, Lask D, Livne PM, Lifshitz DA. Clinical implication of routine stone culture in percutaneous nephrolithotomy-a prospective study. Urology. 2006;67(1):26-9.

21. van Harten RM, Willems RJL, Martin NI, Hendrickx APA. Multidrug-Resistant Enterococcal Infections: New Compounds, Novel Antimicrobial Therapies? Trends Microbiol. 2017;25(6):467-79.

22. Mohamed JA, Huang W, Nallapareddy SR, Teng F, Murray BE. Influence of origin of isolates, especially endocarditis isolates, and various genes on biofilm formation by Enterococcus faecalis. Infect Immun. 2004;72(6):3658-63.

23. Fallah F, Yousefi M, Pourmand MR, Hashemi A, Nazari Alam A, Afshar D. Phenotypic and genotypic study of biofilm formation in Enterococci isolated from urinary tract infections. Microb Pathog. 2017;108:85-90.

24. De Lorenzis E, Alba AB, Cepeda M, Galan JA, Geavlete P, Giannakopoulos S, et al. Bacterial spectrum and antibiotic resistance of urinary tract infections in patients treated for upper urinary tract calculi: a multicenter analysis. Eur J Clin Microbiol Infect Dis. 2020;39(10):1971-81.

25. Chen $D$, Zhang $Y$, Huang J, Liang $X$, Zeng $T$, Lan C, et al. The analysis of microbial spectrum and antibiotic resistance of uropathogens isolated from patients with urinary stones. Int $\mathrm{J}$ Clin Pract. 2018;72(6):e13205.

26. Das P, Gupta G, Velu V, Awasthi R, Dua K, Malipeddi H. Formation of struvite urinary stones and approaches towards the inhibition-A review. Biomed Pharmacother. 2017;96:361-70.

27. Aggarwal KP, Narula S, Kakkar M, Tandon C. Nephrolithiasis: molecular mechanism of renal stone formation and the critical role played by modulators. Biomed Res Int. 2013;2013:292953.

28. Singh P, Yadav S, Singh A, Saini AK, Kumar R, Seth A, et al. Systemic Inflammatory Response Syndrome Following Percutaneous Nephrolithotomy: Assessment of Risk Factors and Their Impact on Patient Outcomes. Urol Int. 2016;96(2):207-11.

29. Dogan HS, Guliyev F, Cetinkaya YS, Sofikerim M, Ozden E, Sahin A. Importance of microbiological evaluation in management of infectious complications following percutaneous nephrolithotomy. Int Urol Nephrol. 2007;39(3):737-42. 


\section{Tables}

Table 1: Analysis of bacterial spectrum of urine and stones (All patients, $\mathrm{N}=1055$ )

\begin{tabular}{llll} 
Bacterial classification & Urine & Stone & $\mathbf{P}$ \\
& $\mathbf{N}=\mathbf{2 2 1}$ & $\mathbf{N}=337$ & \\
& $(\%)$ & $(\%)$ & \\
\hline Escherichia coli & $120(54.3)$ & $148(43.9)$ & $\mathbf{0 . 0 1 6 ^ { * }}$ \\
\hline Proteus mirabilis & $17(7.7)$ & $41(12.2)$ & 0.090 \\
\hline Pseudomonas aeruginosa & $13(5.9)$ & $18(5.3)$ & 0.785 \\
\hline Klebsiella pneumoniae & $13(5.9)$ & $10(3.0)$ & 0.090 \\
\hline Enterococcus faecalis & $15(6.8)$ & $10(3.0)$ & $\mathbf{0 . 0 3 3 ^ { * }}$ \\
\hline Enterococcus faecium & $5(2.3)$ & $7(2.1)$ & 1.000 \\
\hline Staphylococcus epidermidis & $2(0.9)$ & $14(4.2)$ & $\mathbf{0 . 0 2 4 ^ { * }}$ \\
\hline Streptococcus agalactiae & $9(4.1)$ & $5(1.5)$ & 0.056 \\
\hline Other bacteria & $24(10.9)$ & $79(23.4)$ & $<0.001^{\text {*** }}$
\end{tabular}

Table 2: Comparison of bacterial spectrum in urine between infectious and non-infectious stones 


\begin{tabular}{|c|c|c|c|}
\hline Bacterial classification & $\begin{array}{l}\text { Infection stones } \\
\mathrm{N}=91 \\
(\%)\end{array}$ & $\begin{array}{l}\text { non-infection stones } \\
\mathrm{N}=130 \\
(\%)\end{array}$ & $\mathbf{P}$ \\
\hline Escherichia coli & $40(44.0)$ & $80(61.5)$ & $0.010^{*}$ \\
\hline Proteus mirabilis & $16(17.6)$ & $1(0.8)$ & $<0.001^{* \star}$ \\
\hline Pseudomonas aeruginosa & $10(11.0)$ & $3(2.3)$ & $0.007^{* *}$ \\
\hline Klebsiella pneumoniae & $7(7.7)$ & $6(4.6)$ & 0.339 \\
\hline Streptococcus agalactiae & $5(5.5)$ & $4(3.1)$ & 0.493 \\
\hline Enterococcus faecalis & $3(3.3)$ & $12(9.2)$ & 0.084 \\
\hline Enterococcus faecium & $1(1.1)$ & $4(3.1)$ & 0.651 \\
\hline Other bacteria & $9(9.9)$ & $26(19.3)$ & $0.043^{*}$ \\
\hline
\end{tabular}

Table 3: Comparison of bacterial spectrum in stones between infectious and non-infectious stones

\begin{tabular}{|c|c|c|c|}
\hline \multirow[t]{3}{*}{ Bacterial classification } & Infection stones & non-infection stones & \multirow[t]{3}{*}{$\mathbf{P}$} \\
\hline & $\mathrm{N}=137$ & $\mathrm{~N}=\mathbf{2 0 0}$ & \\
\hline & $(\%)$ & $(\%)$ & \\
\hline Escherichia coli & $45(32.8)$ & $103(51.8)$ & $0.001^{\star * *}$ \\
\hline Proteus mirabilis & $37(27.0)$ & $3(1.5)$ & $<0.001^{\star * *}$ \\
\hline Pseudomonas aeruginosa & $14(10.2)$ & $4(2.0)$ & $0.001^{\star \star \star}$ \\
\hline Klebsiella pneumoniae & $7(5.1)$ & $3(1.5)$ & 0.097 \\
\hline Staphylococcus epidermidis & $3(2.2)$ & $11(5.5)$ & 0.135 \\
\hline Streptococcus agalactiae & $3(2.2)$ & $2(1.0)$ & 0.400 \\
\hline Enterococcus faecalis & $4(2.9)$ & $6(3.0)$ & 1.000 \\
\hline Enterococcus faecium & $2(1.5)$ & $5(2.5)$ & 0.705 \\
\hline Other bacteria & $22(16.1)$ & $63(31.0)$ & $0.001^{* *}$ \\
\hline
\end{tabular}

* Statistically significant based on chi- square test $(P<0.05)$. 
** Statistically significant based on chi- square test $(P<0.01)$.

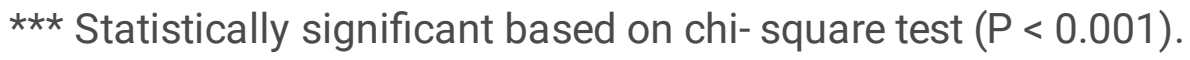

\section{Figures}

a

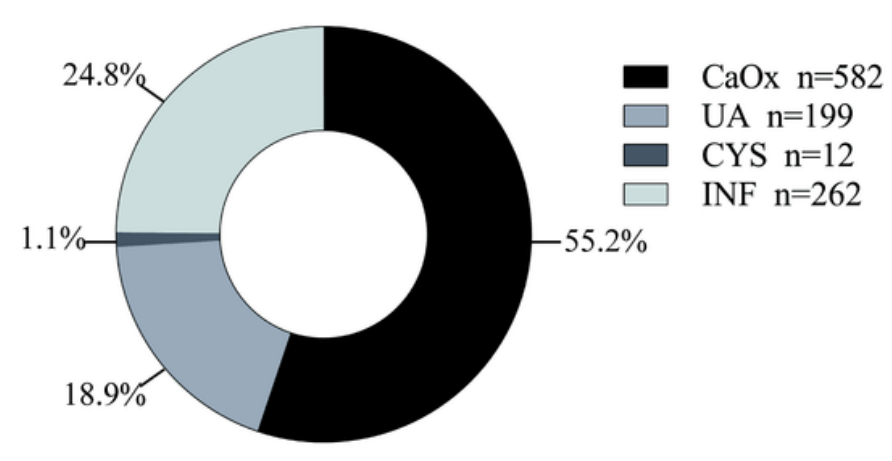

Total $n=1055$ b

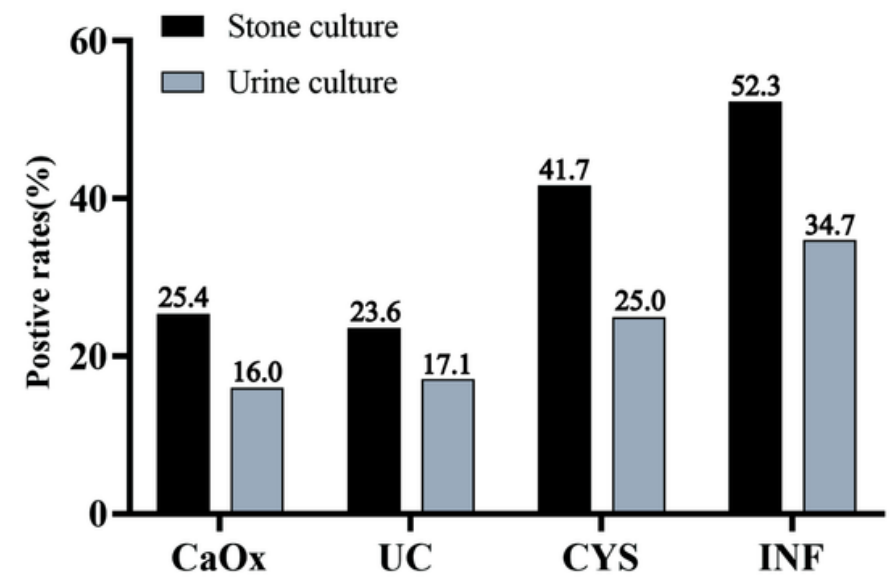

Figure 1

The proportion and positive rate of all stone types. (a) The proportion of four types of stones. CaOx: calcium oxalate stones UA: uric acid stones CYS: cystine stones INF: infectious stones (b) Bacteria detection rate in urine and stones of the four types of kidney stones. 


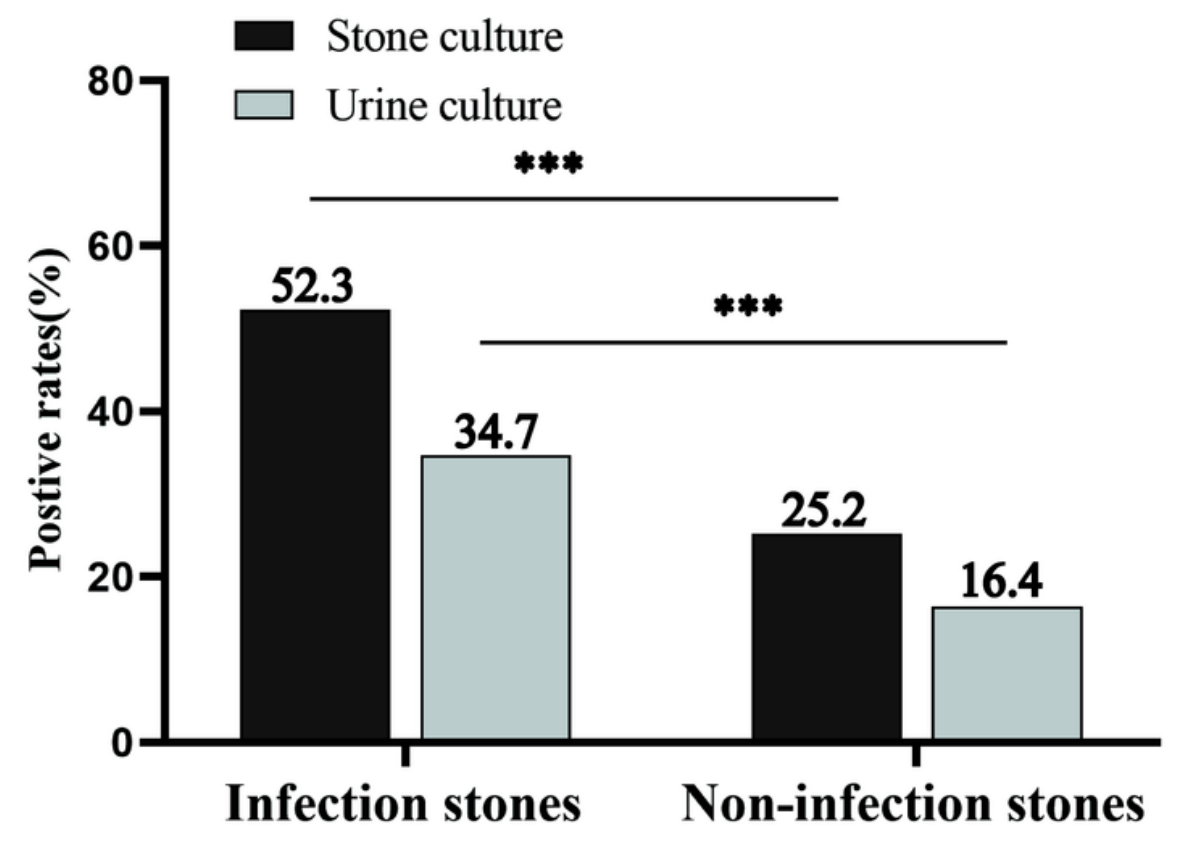

Figure 2

Comparison of the rate of bacteria detection in urine and stones between infection and non-infection stones 
a
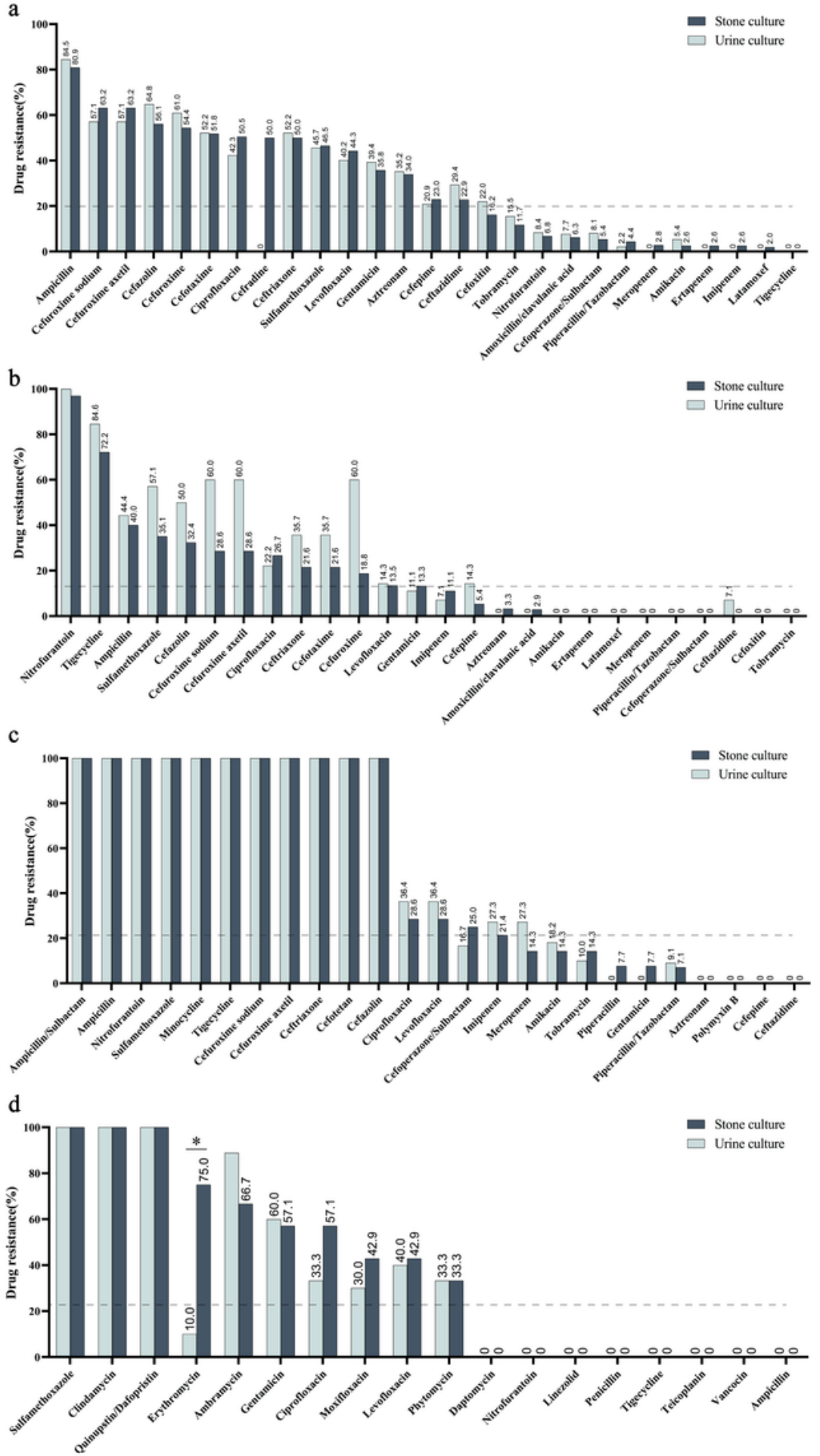

Figure 3

(a): Comparison of antimicrobial resistance rates of E. coli in urine and stones (b): Comparison of antimicrobial resistance rates of Proteus mirabilis in urine and stones (c): Comparison of antimicrobial resistance rates of Pseudomonas aeruginosa in urine and stones (d): Comparison of antimicrobial resistance rates of Enterococcus faecalis in urine and stones *Statistically significant based on chisquare test $(P<0.05)$. 